\title{
Temporomandibular dysfunction syndrome associated with scuba diving mouthpieces
}

\author{
R. S. Hobson MDS, FDS, RCPS \\ Department of Dental Health, Dundee Dental Hospital, Dundee DD1 4HR, UK
}

\begin{abstract}
As previous reports have highlighted that temporomandibular joint dysfunction syndrome can occur during scuba diving due to the use of a diving mouthpiece, 74 divers of varied experience (62 male, 12 female) were asked to evaluate the efficiency of the mouthpiece for the ease of grip, insertion into the mouth, clearing of water, air sharing, comfort and overall efficiency. They also recorded the level of muscle and joint discomfort experienced during diving and non-diving activities. The results indicate that temporomandibular joint problems unrelated to diving are compounded by the use of a diving mouthpiece. The diver's assessment of muscle tension and comfort while using the mouthpiece was found to be a good predictor of whether temporomandibular dysfunction would occur and the assessment scores have been used in a formula to aid selection of a mouthpiece.
\end{abstract}

Keywords: Scuba diving, temporomandibular joint dysfunction

The use of self-contained underwater breathing apparatus (SCUBA) has led to scuba diving becoming one of the most rapidly growing adventure sports, with over 50000 active divers in the $\mathrm{UK}^{1}$. Most diving-related research has been primarily concerned with the physiological effects of hyperbaric conditions in order to achieve longer and deeper dives for the professional diver, and to investigate the effects of prolonged hyperbaric conditions upon the body.

More recently, attention has focused on the problems encountered mainly by sports divers associated with the design of scuba equipment. This equipment provides air on demand (when the diver inhales) by reduction of high pressure air stored in a cylinder worn on the diver's back. The air supply is fed to the diver via a flexible hose which terminates in a mouthpiece held in place by the diver's teeth. The lips form at airtight seal around an extended intraoral labial flange of the mouthpiece, thus excluding water. It is the mouthpiece design which has been implicated in the problems encountered by the sports diver.

A number of papers ${ }^{2-4}$ have described disorders of the temporomandibular joint (TMJ) associated with the use of scuba mouthpieces. Temporomandibular

Address for correspondence: R. S. Hobson, Department of Dental Health, Dundee Dental Hospital, Park Place, Dundee DD1 4HR, Scotland

(C) 1991 Butterworth-Heinemann Ltd.

0306-3674/91/010049-03 joint dysfunction (also known as myofascial pain dysfunction) is a problem occurring in the TMJ and/or its associated musculature. It is due to either an occlusion imbalance or over-exertion of the joint and muscles, resulting in pain.

Pinto $^{3}$ and Roydhouse ${ }^{2}$ have suggested that use of a diving mouthpiece can result in local inflammation of the TMJ, which may result in a blockage of the eustachian tube and possibly lead to labyrinthine dysfunction and associated vestibular disturbances, such as vertigo and disorientation. These are potentially hazardous conditions to occur underwater.

All commercially available diving mouthpieces conform to a similar basic design. A labial flange aids the lip seal and an interdental biting platform is gripped between the diver's teeth. This in nearly all cases involves a forward movement of the mandible enabling the incisor and canine teeth to grasp the mouthpiece. The resulting effect is a lack of posterior support and uneven loading within the TMJ and associated musculature (Figure 1). The human masti-

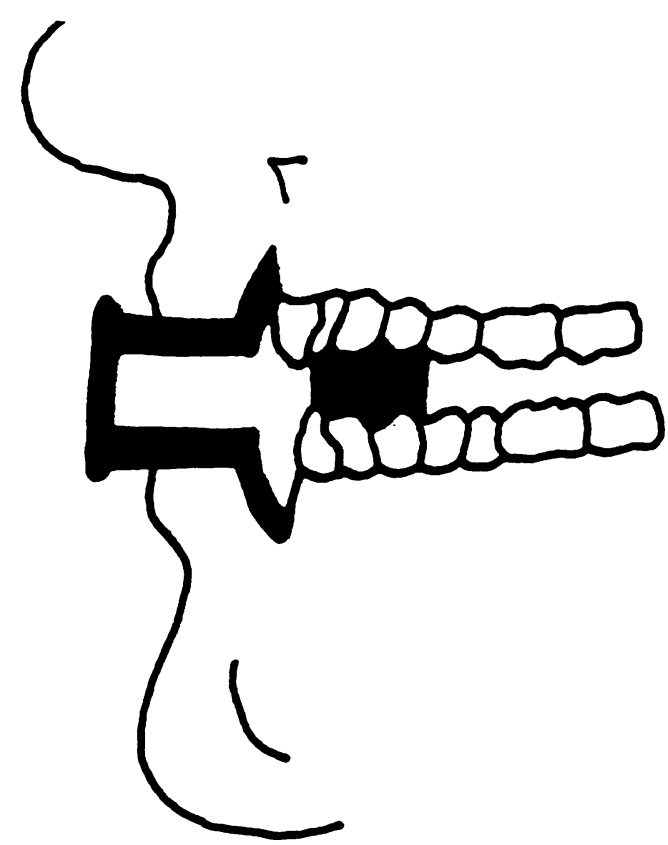

Figure 1. Illustration to show how the position of the bite platform in commercially available mouthpieces (gripped by incisor and canine teeth) results in the lack of posterior occlusal support 
Table 1. Sex and diving experience of sample group

\begin{tabular}{lccc}
\hline & Novice & Intermediate & Experienced \\
\hline Male & 8 & 19 & 35 \\
Female & 2 & 4 & 6 \\
\hline
\end{tabular}

catory system is both anatomically and functionally unsuited to cope with the extended periods of isometric muscle contraction required to retain a scuba mouthpiece in this abnormal jaw position and it is easy to envisage why TMJ problems occur during its use.

The incidence of TMJ disorders among divers has been reported as $24 \%$ by Roydhouse ${ }^{5}$. More recently Hobson $^{6}$ reported that $68 \%$ of dental pain associated with scuba diving was due to TMJ dysfunction, the pain being severe enough on occasions to abandon the dive.

A survey was undertaken to ascertain the important factors in diving-related TMJ problems in order that guidance might be given in the selection of a mouthpiece.

\section{Material and methods}

A total of 74 divers (62 male and 12 female) of varied experience (Table 1) were asked to complete a questionnaire, consisting of seven direct questions, to indicate the efficiency of their mouthpieces. They were also asked whether they experienced TMJ pain during the use of a diving mouthpiece and if they experienced TMJ dysfunction during non-diving activities. The manufacturer of the scuba equipment and mouthpiece was recorded, and additional space was provided for further comment and description.

They were asked to score out of $10(0=$ awful and $10=$ perfect) for ease of grip, insertion of the mouthpiece into the mouth, water clearing (the action of clearing a waterlogged mouthpiece underwater), air sharing (an emergency procedure where two divers breathe alternatively from the same mouthpiece), comfort during use and their judgement of the overall efficiency of the mouthpiece. The level of muscle/TMJ discomfort was also scored out of 10 (10 = excruciating pain and $0=$ no pain). The frequency of $\mathrm{TMJ} / \mathrm{muscle}$ pain during diving and on other occasions was recorded, the site of pain being indicated on diagrams of the face, including right and left profiles.

\section{Results}

The data were collated and analysed on an IBM microcomputer using a statistical analysis package (Statsgraphics, Rockville, MD 20852, USA).

The possible relationship between divers' suffering TMJ pain during diving and non-diving activities was tested using the Kendal rank correlation. This gave a result of $0.2698(P=0.0003)$. This indicates that those divers who suffer TMJ dysfunction during non-
Table 2. Table mouthpiece versus pain

\begin{tabular}{lrcr}
\hline $\begin{array}{l}\text { Manufacturer of } \\
\text { mouthpiece }\end{array}$ & Pain & No pain & $\begin{array}{l}\text { Incidence } \\
\text { of pain } \\
(\%)\end{array}$ \\
\hline US Divers & 12 & 5 & 70 \\
Scuba-Pro & 9 & 1 & 90 \\
Spiro & 6 & 4 & 60 \\
Kawasaki & 4 & 4 & 50 \\
Posidon & 6 & 0 & 100 \\
Customised & 7 & 0 & 100 \\
Miscellaneous & 11 & 5 & 69 \\
\hline
\end{tabular}

Customised = modification of any brand or an individually constructed mouthpiece

diving activities are extremely likely to experience the same problem whilst diving as a result of using a mouthpiece.

In order to investigate whether individual makes of mouthpieces were more likely to cause TMJ dysfunction than others, the effect of each brand on pain was tested by cross tabulation (Table 2): the resulting difference, measured by $\chi^{2}$, was not significant $(P=$ $0.13)$. However, the number within some of the groups was small for accurate testing. However, certain mouthpieces (Posidon, Scuba-Pro, Customised) seem to have a higher incidence of TMJ pain than other makes (Table 2).

In order to ascertain which variables divers thought were the best predictors of TMJ pain during the use of a diving mouthpiece, discriminant analysis was used. This has the ability to test for the accuracy of a variable or group of variables in predicting a given result.

Initially a combination of all the variables (ease of grip, insertion of mouthpiece, water clearing, air sharing, comfort, muscle tension) were tested against the incidence of TMJ pain during diving, giving an accuracy of $63.5 \%$ for pain prediction. This indicates that those divers who gave a low score for the majority of the variables, were predicted to have TMJ pain in approximately six out of ten cases. Analysis using only the variables comfort and muscle tension improved this to a $71.1 \%$ accuracy for pain prediction, i.e. the addition of the other variables decreased the accuracy of pain prediction. The unstandardized discriminant function coefficients show that muscle tension with a value of 0.262 is more important than comfort $(-0.159)$ in the prediction of TMJ pain occurrence. However, the use of either muscle tension or comfort on their own reduced the accuracy to less than $70 \%$ (muscle tension 65.8\%; comfort $60.2 \%$ ). The combination of assessment of muscle tension and comfort by the diver produced the most accurate prediction of TMJ dysfunction during the use of a particular mouthpiece.

The use of discriminant analysis allows the construction of a formula for the prediction of diving pain, which the diver may use in order to assess the most suitable mouthpiece. The diver, having used the mouthpiece, gives a score out of 10 for comfort $(0$ $=$ awful, $10=$ perfect) and for the level of muscle 
tension $(0=$ none, $10=$ excruciating $)$ and places it in the formula:

$($ Muscle tension score $\times 0.26)-($ Comfort score $\times 1.16)=Z$

If the $Z$ value is positive there is a $70 \%$ possibility of pain, and if the $Z$ value is negative there is a $70 \%$ possibility of being pain free. If the diver finds that he or she has similar results for a number of mouthpieces then the mouthpiece with the lowest muscle tension score should be the better prospect for the prevention of TMJ pain.

\section{Discussion}

The finding that non-diving TMJ pain is exacerbated by the use of a diving mouthpiece has previously been noted by Roydhouse ${ }^{2}$. It is obvious that if a diver already suffers from TMJ problems then the use of a diving mouthpiece of unsuitable design is likely to aggravate the problem. Goldstein and $\mathrm{Katz}^{7}$ and Mack et al. ${ }^{8}$ have investigated factors in mouthpiece design and have proposed improved designs which reduce the incidence of TMJ dysfunction. Unfortunately, at present, these designs are not commercially available.

That there was no significant difference between makes of mouthpiece and the incidence of TMJ pain may be due to the size of the mouthpiece groups, and the similarity of the commercial designs available. This appears to be at variance with the findings of Ingervall and Warfvinge ${ }^{9}$ who showed, using electromyography (EMG), that certain mouthpieces were more likely than others at creating increased muscle tension, and that a customised mouthpiece gave the lowest EMG activity. Mack et al. ${ }^{8}$ found that mouthpieces which reduced discomfort varied significantly in design from those commercially available, having a thinner bite platform placed between premolar and molar teeth.

It is interesting that all divers using Posidon or Customised mouthpieces reported TMJ pain during diving. The Posidon design differs from most other commercially available mouthpieces in that it is of hard rubber construction with thicker bite blocks placed between the canine teeth. This is in direct contrast with the recommendations by Goldstein and $\mathrm{Katz}^{7}$ and Mack et al. ${ }^{8}$ that mouthpieces should be constructed of a resilient material, e.g. silicone. Unfortunately it was not possible to examine those mouthpieces that had been customised to ascertain how they varied from commercial designs. It could be surmised that these divers were already suffering from significant TMJ problems, which led them to modify their mouthpiece in an attempt to alleviate their discomfort.
The results indicate that the diver's assessment of muscle tension and comfort, during use of a mouthpiece, is the best indicator of diving TMJ pain. The other factors implicated in mouthpiece design (ease of grip, insertion into the mouth, water clearing) by Mack et al. ${ }^{8}$ do not improve the accuracy of TMJ pain prediction. This may be due to these factors, although necessary for the safe and efficient function of a diving mouthpiece, being regarded as less important by the diver. The exercises of water clearing and air sharing are very rarely undertaken during the course of normal diving.

The design of commercially available mouthpieces seems to fall short of those recommendations of Goldstein and Katz ${ }^{7}$ and Mack et al. ${ }^{8}$ Certain designs appear to be better in preventing TMJ pain. The diver, whilst choosing his equipment, would be well advised to undertake the trial of a number of mouthpieces in order to find the design with the least likelihood of causing TMJ dysfunction, and not just accepting the mouthpiece supplied. Ingervall and Warfvinge ${ }^{9}$ and Mack et al. ${ }^{8}$ have suggested that any trial of a mouthpiece should involve a period of use of not less than $15 \mathrm{~min}$ duration, with a rest period of similar time before assessment of the next mouthpiece.

A diver with a diving-related TMJ problem should seek dental help in the construction of a customised mouthpiece or undertake a trial of a number of commercially available mouthpieces. Use of the formula for assessment should indicate the mouthpiece most likely to relieve his or her symptoms.

\section{Acknowledgement}

The author thanks Mr D.R. Stirrups for advice and help with statistical analysis, and Mrs C. Barr for typing the manuscript.

\section{References}

1 Annual Report and Accounts. British Subaqua Club, 1989.

2 Roydhouse N. The jaw and scuba diving. J Otolaryngol Soc Australia 1977; 4: 162-5.

3 Pinto OF. Temporomandibular joint problems in underwater activities. J Prosthet Dent 1966; 16: 772-81.

4 Storer $\mathrm{R}$ and Bowman $\mathrm{A}$. An unusual factor in disharmony of the masticatory system. Br Dent J 1969; 126: 80-1.

5 Roydhouse N. 1001 disorders of the ear, nose and sinuses in scuba divers. Can J Appl Sport Sci 1985; 10: 99-103.

6 Hobson RS. Diving and dental pain. S Pac Underwater Med Soc J 1987; 17: 43-6.

7 Goldstein GR, Katz W. Divers' mouth syndrome. NY State Dent J 1982; 10: 523-5.

8 Mack PJ, Hobson RS, Askell J. Dental factors in scuba mouthpiece design. Br Dent J 1985; 158: 141-2.

9 Ingervall B, Warfvinge J. Activity of orofacial musculature during use of mouthpieces for diving. J Oral Rehabil 1978; 5: 269-77. 